\title{
Cooperative Cloud Service Aware Mobile Internet Coverage Connectivity Guarantee Protocol Based on Sensor Opportunistic Coverage Mechanism
}

\author{
Qin Qin, ${ }^{1}$ Yong-qiang $\mathrm{He},{ }^{1}$ and Li-ming $\mathrm{Nie}^{2}$ \\ ${ }^{1}$ College of Computer, Henan Institute of Engineering, Zhengzhou 450007, China \\ ${ }^{2}$ School of Software Technology, Dalian University of Technology, Dalian 116621, China \\ Correspondence should be addressed to Qin Qin; qq@haue.edu.cn
}

Received 20 August 2015; Revised 30 November 2015; Accepted 9 December 2015

Academic Editor: James Nightingale

Copyright (C) 2015 Qin Qin et al. This is an open access article distributed under the Creative Commons Attribution License, which permits unrestricted use, distribution, and reproduction in any medium, provided the original work is properly cited.

In order to improve the Internet coverage ratio and provide connectivity guarantee, based on sensor opportunistic coverage mechanism and cooperative cloud service, we proposed the coverage connectivity guarantee protocol for mobile Internet. In this scheme, based on the opportunistic covering rules, the network coverage algorithm of high reliability and real-time security was achieved by using the opportunity of sensor nodes and the Internet mobile node. Then, the cloud service business support platform is created based on the Internet application service management capabilities and wireless sensor network communication service capabilities, which is the architecture of the cloud support layer. The cooperative cloud service aware model was proposed. Finally, we proposed the mobile Internet coverage connectivity guarantee protocol. The results of experiments demonstrate that the proposed algorithm has excellent performance, in terms of the security of the Internet and the stability, as well as coverage connectivity ability.

\section{Introduction}

With the development and integration of wireless network, mobile communication [1], sensor [2], cloud platform, and so forth, the next generation of Internet has been widely used and developed in the field of real-time monitoring, full coverage of mobile network, and poor environment data acquisition and communication. The mapping of Internet nodes and sensor nodes establish the heterogeneous communication, and provide support for the monitoring of information storage and forwarding and real-time processing. However, because of the diversity of environmental monitoring needs and the forwarding of sensor information collection and the network coverage and connectivity [3] of all kinds of bad environment, it is still a hot and key issue of the next generation Internet.

There are some researches of Internet coverage connectivity. A coverage-based hybrid overlay was proposed in article [4], which disseminates messages to all subscribers without uninterested nodes involved in and increases the average number of node connections slowly with an increase in the number of subscribers and nodes. A new notion of intermittent coverage for mobile users was introduced in article [5], which provides worst-case guarantees on the interconnection gap, the distance, or expected delay between two consecutive mobile-AP contacts for a vehicle. Efficient node coverage scheme was proposed for addressing the scheduling issue of nodes of the underground space near surface [6]. The authors of article [7] proposed the supporting plant designer during wireless coverage prediction, virtual network deployment, and postlayout verification sensor opportunistic coverage mechanism. A low-cost way [8] was proposed for public transit operators to enhance quality of experience for passengers who access the Internet. The control flow graph and cyclomatic complexity of the example program [9] were used to find out the number of feasible paths present in the program and compared it with the actual number of paths covered by genetic algorithm. 
About the coverage issue of wireless sensor networks (WSNs), Zhao et al. [10] analyzed the changes of opportunistic coverage ratios. The new cooperative opportunistic fourlevel model for IEEE 802.15.4 wireless personal area network was proposed by Rohokale et al. [11]. A localization scheme named Opportunistic Localization by Topology Control was proposed in [12] for sparse Underwater Sensor Networks. The use of an MAS as an appropriate mechanism was advocated by different stakeholders in [13].

For studying the relationship of cloud platform and coverage issue of Internet, a service aware location update mechanism was proposed in [14], which can detect the presence and location of the mobile device. A novel quality aware computational cloud selection service was proposed and evaluated in [15]. The cost and energy aware serviceprovisioning scheme [16] was presented for mobile client in mobile cloud, which includes two-stage optimization process. A novel disaster-aware service-provisioning scheme was proposed in [17], which multiplexes service over multiple paths destined to multiple servers/datacenters with many casting. A privacy-aware cross-cloud service composition method was proposed in [18] based on its previous basic version HireSome-I.

However, these research results ignored the resources management issue of cloud platform and the mobile Internet requirements and had a little research of coverage guaranteeing ability of WSNs. Then, we proposed the cooperative cloud service aware mobile Internet coverage connectivity guarantee protocol based on sensor opportunistic coverage mechanism.

The rest of the paper is organized as follows. Section 2 gave the sensor opportunistic coverage mechanism. In Section 3, we proposed the model of cooperative Cloud service aware. The mobile Internet coverage connectivity guarantee protocol was proposed in Section 4. The results of the mathematical analysis and simulation verification are given in Section 5. Finally, we conclude the paper in Section 6.

\section{Sensor Opportunistic Coverage Mechanism}

In the context of mobile Internet, how to deploy wireless sensor networks to achieve reliable and stable network coverage has become the key technology of wireless sensor networks and the Internet. Separate wireless sensor network coverage rule is specified based on different type of structure. Generally, the same wireless sensor network only includes a single cover rule or a geometric figure. This is not conducive to the combination of large scale sensor networks and mobile Internet. In order to meet the needs of the integration of the Internet and reduce the complexity and robustness of wireless sensor network coverage control, a mobile Internet based on sensor deployment and network coverage algorithm is proposed. The algorithm can ensure the distributed computing and the full connected routing of sensor nodes. The algorithm can be implemented based on the irregular coverage of mobility.

Based on the driving of Internet mobile node, the algorithm combines the opportunity dynamic coverage rule and communication between the mobile node and the sensor node to achieve a high reliability and real-time security.

$N$ denotes the number of mobile nodes which are used to connect the sensor network with the Internet. Wireless sensor networks (WSNs) deployment node number is $M$. The nearest distance between the mobile node and the sensor node is $d_{0}$. The minimum distance between the sensor nodes is $d_{1}$. In order to reduce the impact of the Internet node's mobility on sensor network coverage, the deployment distance parameters need to meet the following formula:

$$
\frac{S\left(d_{\max }\right)}{\sum_{i=1}^{M} d_{1}^{i}} \leq \frac{\operatorname{MI}\left(d_{\max }\right)}{\sum_{j=1}^{N} d_{0}^{i}}
$$

Here, $S\left(d_{\max }\right)$ denotes the maximum connection distance of sensor nodes in WSNs. $\mathrm{MI}\left(d_{\max }\right)$ denotes the maximum connection distance between Internet and WSNs.

At the same time, in order to ensure the deployment of sensor nodes independently, the communication distance between adjacent sensor nodes and the Internet mobile node should be in accordance with formula (2), which is used to ensure that the mobile node and sensor nodes connected with independent characteristics

$$
\frac{\operatorname{MI}\left(d_{\max }\right)}{v_{\max }} \leq t_{0}\left[\frac{\mu \cos \alpha}{e^{-S\left(d_{\max }\right)}}\right]^{N / M}
$$

Here, $v_{\max }$ is the maximum moving speed of Internet node. $t_{0}$ is the delay of transmitting signal from Internet node to sensor node. $\mu$ denotes the opportunistic connection ratio between Internet node and sensor node. $\alpha$ is the angle of the antenna direction of the Internet node and the sensor node direction.

Sensor node $S_{i}$ can sense the Internet node $\mathrm{MI}_{i}$. Internet nodes help sensor nodes to establish network coverage with strong connectivity. The opportunity coverage ratio is shown in the following formula:

$$
\begin{aligned}
C_{(S, \mathrm{MI})} & = \begin{cases}0, & t \geq t_{\mathrm{TH}} \\
1, & t \leq t_{\mathrm{TH}},\end{cases} \\
t_{\mathrm{TH}} & =\mu\left(\frac{d_{(S, \mathrm{MI})}}{v_{\mathrm{MI}}}\right)^{\delta} .
\end{aligned}
$$

Here, $C_{(S, M I)}$ denotes the connection ratio between nodes. $t_{\mathrm{TH}}$ is the maximum delay of keeping communication. $\delta$ is the probability of occurrence and transformation of Internet node movement direction.

The mobile nodes and the sensor nodes would use signal level cooperation communication. The communication between Internet mobile node MI and the $m$ sensor nodes would create the neighbor coverage relationship. They are common sensing coverage and control an area, which are shown in Figure 1. The opportunistic coverage strength OCI 


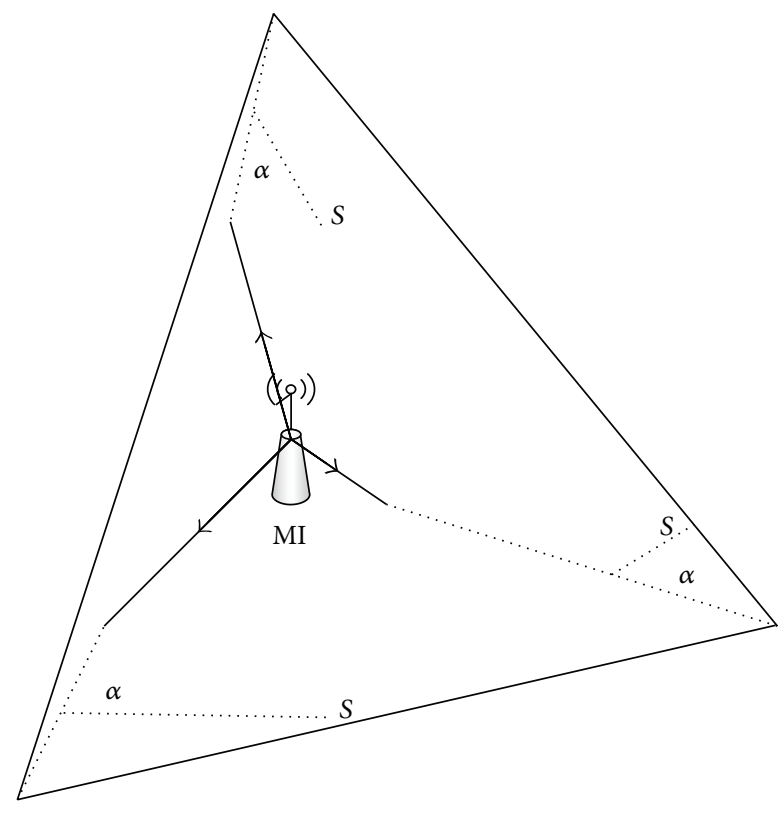

FIGURE 1: Common sense and coverage control area.

between sensor node and the mobile node can be obtained by the following formula:

$$
\begin{gathered}
\text { OCI }=|\gamma-\mu|^{2} \sum_{i=1}^{m} C_{i, \mathrm{MI}}, \\
\gamma=\frac{\sum_{i=1}^{m} t_{i}}{t_{\mathrm{TH}}}
\end{gathered}
$$

Here, $\gamma$ is the probability of connecting time greater than $t_{\mathrm{TH}}$ between the Internet nodes and sensor nodes.

Through the combination of the Internet node deployment area and sensor network deployment area of the mobile Internet, the opportunity to cover the intensity MI-OCI can be obtained through the node between the opportunity connection and collaborate sensing, as shown in the following formula:

$$
\begin{aligned}
\operatorname{MI}-\operatorname{OCI}(N, M) & =\frac{\sqrt{t_{\mathrm{TH}}\left|v_{\max }-v_{\min }\right|^{2}} \sum_{i=1, j=1}^{N, M} d_{i, j}}{\mu|\gamma-\delta|^{2} v_{\mathrm{TH}}}, \\
v_{\mathrm{TH}} & =\frac{\sqrt{\left|S\left(d_{\mathrm{max}}\right)^{2}-\mathrm{MI}\left(d_{\max }\right)^{2}\right|}}{t_{\mathrm{TH}}} .
\end{aligned}
$$

Here, $v_{\mathrm{TH}}$ is the Internet node moving speed threshold.

\section{Cooperative Cloud Service Aware Model}

With the rapid development of the Internet and WSNs, the next generation network convergence and cloud platform business should be improved. So, the cloud platform service business platform is proposed. The service platform has the following characteristics.
(1) To guide the deployment, implementation, and management of WSNs is the center of service support and user requirements.

(2) Data services and forwarding services are capable of supporting multinetwork integration and heterogeneous services and are not independent of the front-end device and forwarding gateway for specific wireless sensor networks.

(3) The size of the cloud platform has a dynamic adjustment ability. According to different network traffic and forwarding data content, the cloud devices have the way of cooperation between the management and control.

(4) The information service quality and wireless sensor network resource utilization can be perceived as an open and reliable access to provide services to the front of the Internet and the sensor.

The architecture model of the cloud platform service business platform is shown in Figure 2. Cloud service business support platform of the Internet application service management capabilities and wireless sensor network communication service capabilities are abstracted as the architecture of the cloud support layer. The support layer is a business entity shared by all cloud services. Cloud service middleware platform provides the deployment and execution environment for all kinds of Internet applications and data services for wireless sensor networks. Cloud physical layer for the Internet and WSNs could provide the core support of various front-end equipment and services and cross layer interface.

The above architecture shows that the perception of cloud services has important significance for the Internet management and WSNs coverage, as well as topology management. Therefore, the application of the Internet could be encapsulated as cloud services. Various types of sensor frontend devices and cloud services could be considered as the perception of objects, through the aggregation of the Internet service and WSNs, which is used to provide a basis for the internet.

The cloud service perception model is shown in Figure 3. Based on the Internet mobility management business security mechanism and the WSNs connectivity guarantee mechanism, the perception model is proposed. The complexity of cloud computing through WSNs communication between different devices could be reduced. Cloud platform through cooperative cloud services for WSNs deployment and coverage could provide a unified positioning business. Cloud platform through the cooperative cloud service could be packaged in the cloud platform middleware layer. Web services of the Internet and WSNs data collection and forwarding services could be provided by the combination of cooperative cloud aggregation services. This service can provide reference information and decision basis for route maintenance and cooperative coverage in the WSNs coverage area. In particular, there are the perception pile between the Internet and WSNs, which is composed of wireless multiple word system bus. In order to improve the parallelism of the system, we designed the control feedback interaction 


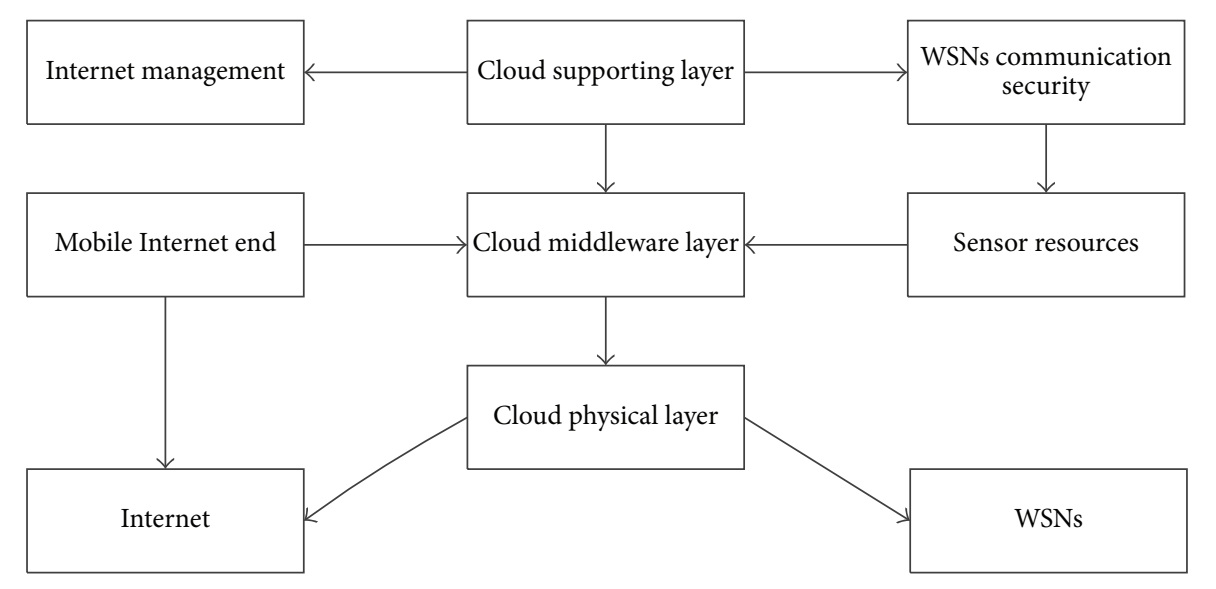

Deployment connected coverage

FIGURE 2: Architecture model of cloud service platform.

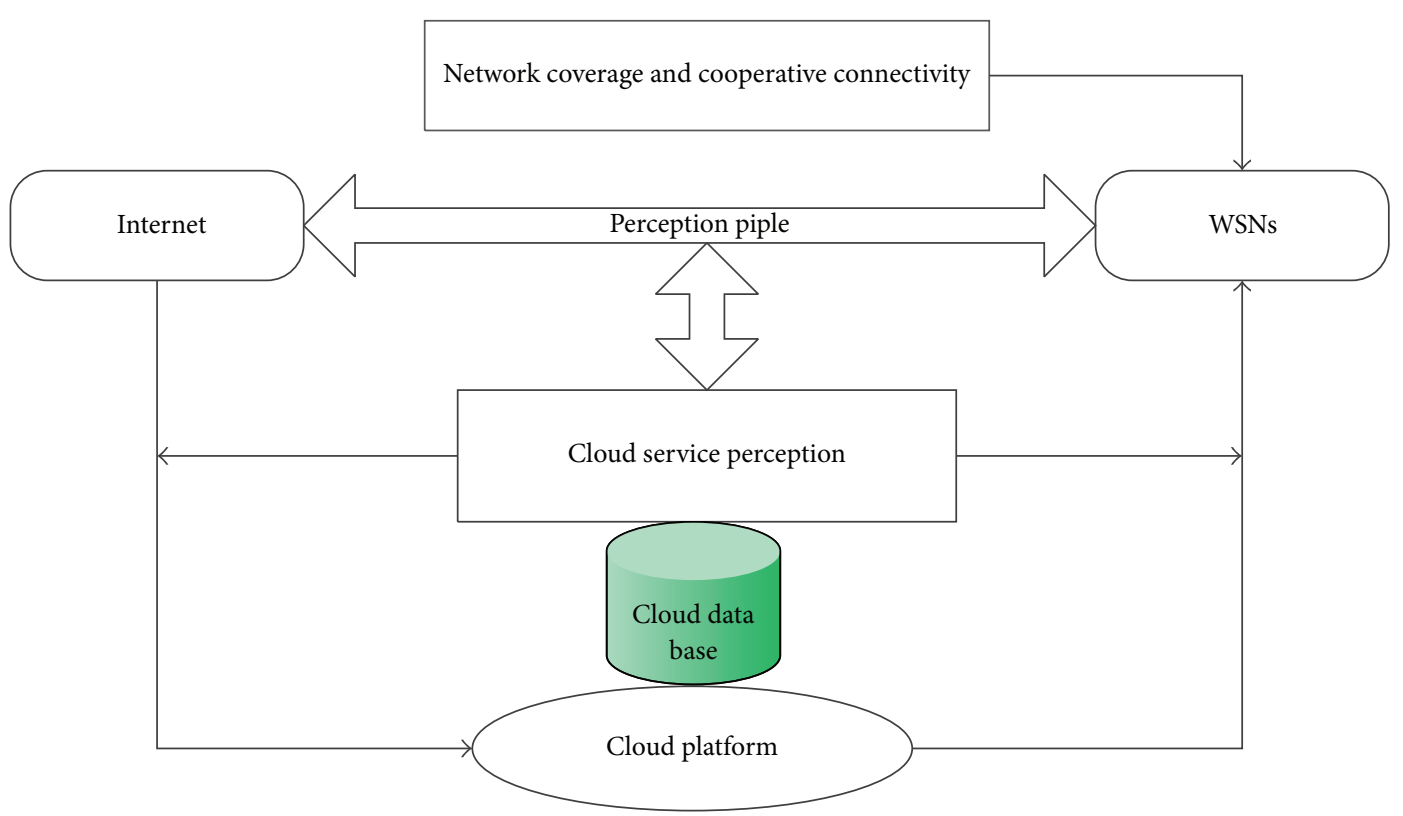

FIGURE 3: Cloud service perception model.

line, which is from cloud service perception platform to the Internet and WSNs.

\section{Mobile Internet Coverage Connectivity Guarantee Protocol}

Mobile Internet applications can achieve the application development through the integration of cloud platforms and WSNs. When the application is extended, the problem of mobile management and resource management and reliable information forwarding should be considered. The high coverage ratio could be maintained by deploying a large number of redundant devices or sensor nodes in the cloud platform and WSNs. But about the Internet node service life, WSNs sensor node resource management and cloud computing scheduling, and so forth, the network connectivity could not kept by increasing the size of equipment.

Therefore, it is the key technology to make full use of the opportunity of each sensor node in wireless sensor network, and the effective driving ability of the cloud platform service, which is used to ensure the Internet connectivity. Through the WSNs, the sensor nodes and the Internet nodes are in the mobile state, which can reduce the resource consumption and realize the effective seamless coverage of the network. In order to reduce the impact of the mobile Internet connectivity coverage, the Internet and the cloud platform play a role in the root node of the cloud device. The diffusion of Internet nodes and sensor nodes coverage can be opportunistic, dynamic, and adjusted at the same time to achieve maximum coverage of connectivity and through collaborative optimization control to achieve global connectivity. This can reduce 
the resource consumption of each node and can reduce the probability of the occurrence of invalid overlap between nodes, as shown in the following formula:

$$
\begin{aligned}
C_{\mathrm{RC}} & =\sum_{k=1}^{N+M} R_{k} \frac{S\left(d_{\max }\right)+\mathrm{MI}\left(d_{\max }\right)}{\sum_{i=1}^{M} d_{1}^{i} d_{0}^{i}}, \\
P_{\mathrm{IO}} & =\sum_{i=1}^{C_{N}} \rho_{i} \frac{\sqrt{\left|\mathrm{SC}_{N}-\mathrm{SC}_{M}\right|}}{\mathrm{SC}_{N+M}} .
\end{aligned}
$$

Here, $C_{\mathrm{RC}}$ denotes cover resource consumption. $R_{k}$ denotes the resource consumption of node. $P_{\mathrm{IO}}$ is the probability of invalid overlap. $C_{N}$ is the cloud number of the platforms. $\rho$ denotes the active ratio of cloud. SC denotes the covering area.

Based on the Internet coverage mechanism and the cooperative security mechanism, the following problems should be considered.

(1) When the mobile node and sensor nodes are used in the model, the sensing distance of the node is related to the computing power of the cloud computing power. According to the node sensing distance, the Internet coverage is irregular geometry area composed by a number of mobile nodes and sensor nodes. Internet coverage area is not affected by node mobility.

(2) The sensor covers the sensing area opportunistically and deploys sensor nodes based on the remaining resources of the Internet node and cloud service needs.

(3) When the sensor network is deployed by the terrain or the impact of a large building, it can be through the cloud platform of collaborative cloud service sensing mechanism, the communication of all nodes to the same plane, as shown in Figure 4.

(4) The hierarchical communication architecture of sensor nodes and the hierarchical protocol of Internet nodes occur through the cloud platform to achieve cross layer interaction. The nodes and the cloud devices in the Internet are covered by the Internet, which can communicate directly with the sensor nodes and the cloud devices, and the heterogeneous communication protocols can be handled transparently.

The WSNs opportunity coverage role of Internet mobile coverage connectivity is reflected in the following aspects.

(1) When the sensor nodes have separated from the Internet, these nodes would broadcast a request of the subnetwork separation and reconstruct to the neighbor nodes. After receiving the request, the nodes are calculated by the cloud platform to maintain the network connectivity. If you cannot meet the needs of the Internet seamless coverage, the cloud platform issued a collaborative cloud service perception control in the Internet node and sensor nodes to

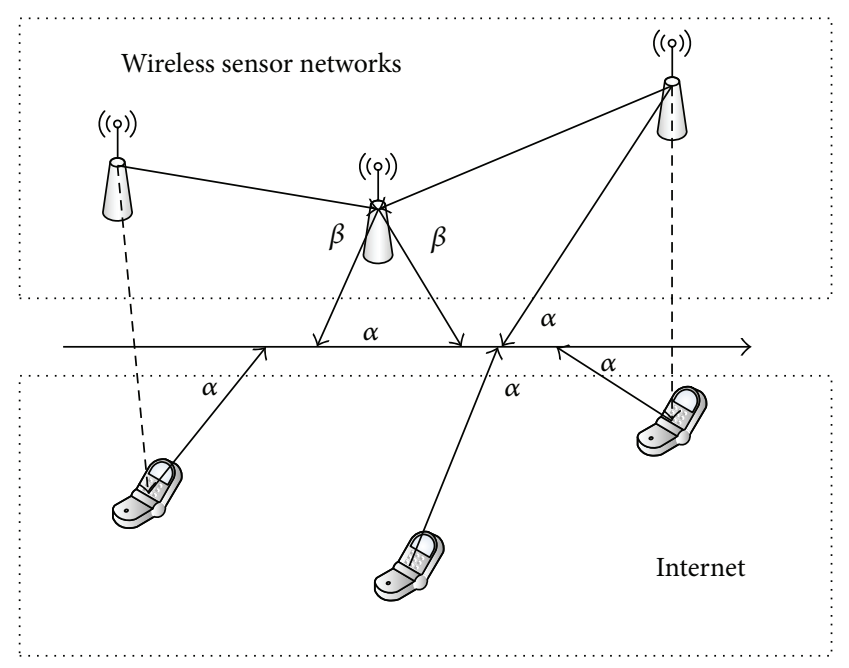

FIGURE 4: Communications mapping with cooperative cloud aware scheme.

search for collaborative gaps and opportunity to drive the region for ensuring the full connectivity of the Internet monitoring area and real-time coverage, as shown in the following formula:

$$
\begin{aligned}
d_{\max (S, \mathrm{MI})} & =\sqrt{\left|d_{\max (S)}-d_{\min (\mathrm{MI})}\right|^{2}} \sum_{i=1, j=1}^{N, M} d_{i, j}, \\
\mathrm{SC}_{\text {crevice }} & =\frac{\sqrt{\left|\mathrm{SC}^{2}{ }_{N}-\mathrm{SC}^{2}{ }_{M}\right|}}{\tan \left(d_{\max (S, \mathrm{MI})} / \theta\right)} .
\end{aligned}
$$

Here, $\theta$ is the Internet node and sensor node antenna angle. $\mathrm{SC}_{\text {crevice }}$ is the gap area.

(2) In order to avoid the loss caused by the network nodes and sensor nodes due to overlapping coverage area, the cloud platform and data forwarding provide seamless coverage and full connected routing. We must gradually reduce the communication delay between the nodes in the coverage area, so that the three-party interaction and coverage area of the cloud platform, the Internet, and WSN achieve the best equilibrium state.

(3) When the channel quality is good, it can increase the share of the three parties, the largest consumption of resources to achieve maximum coverage area. When the channel quality is poor, the sensor can increase the opportunity to cover the weight and the cloud service cooperative sensing coefficient and achieve the optimal control in the area of Internet coverage and connectivity.

\section{Mathematical Analysis and Simulation Verification}

The performance of the proposed algorithm is analyzed in this section, which includes the stability, connectivity, and 


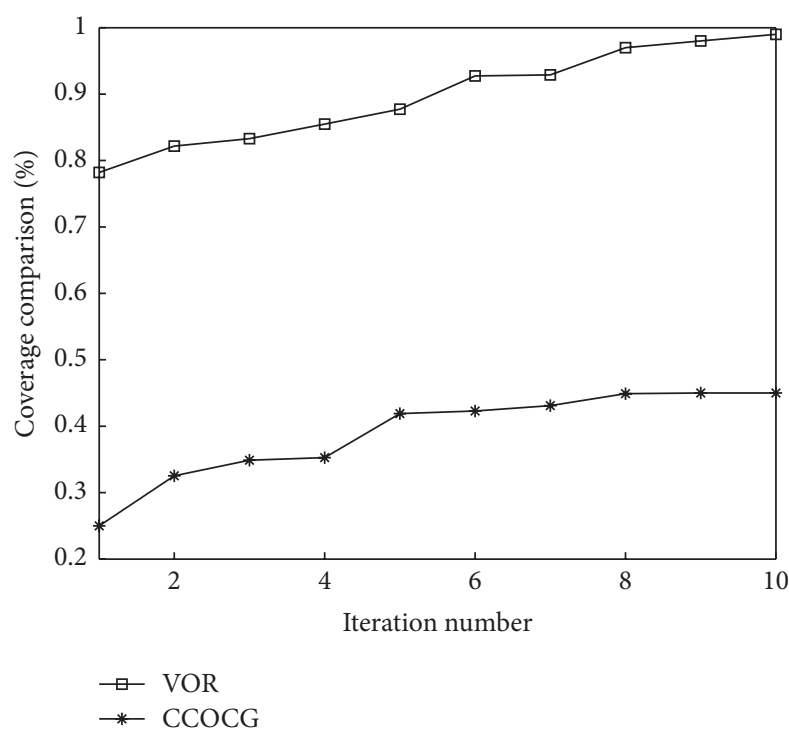

(a) $N=15, M=15$

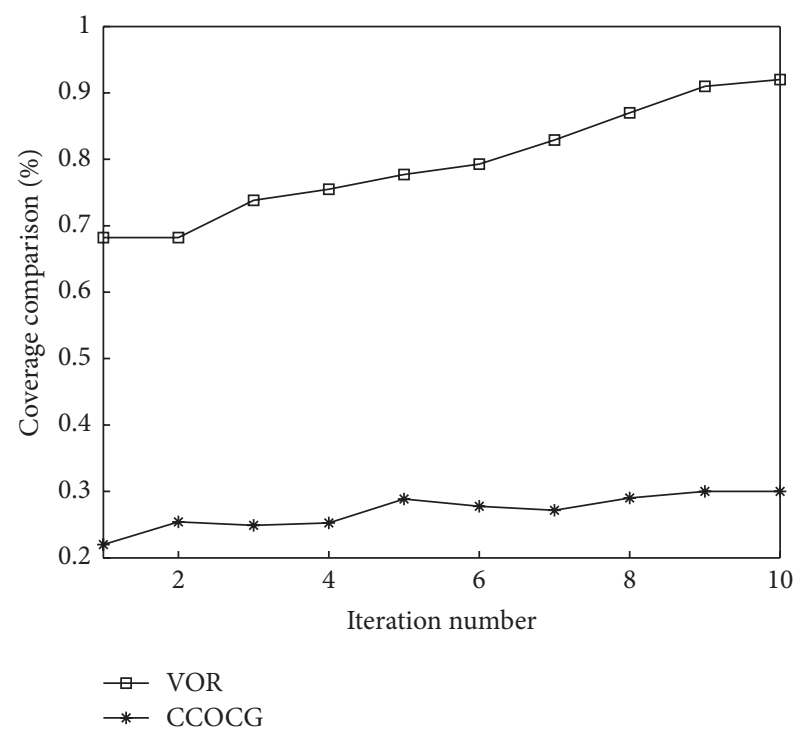

(b) $N=20, M=10$

FIGURE 5: Coverage comparison.

security of the Internet. The coverage stability of the Internet is considered, both the Internet node and the sensor node sensing area coverage and the connectivity security capability. When the security capability is strong, the security time of the proposed algorithm is investigated, including the Internet node iteration time, the cloud computing time, and the forwarding time of the sensor. The time spent on the Internet is determined by the time of the node moving speed, cloud computing delay, and sensor node coverage.

The cost of Internet connectivity includes:

(1) the network nodes in order to maintain the loss of the mobile speed;

(2) the total number of nodes required by the Internet;

(3) the resource consumption of the cloud devices to maintain continuous calculation;

(4) the energy consumption of sensor nodes and the energy consumed in communication.

So, by comprehensive evaluation of coverage connectivity and security costs, we can better analyze the performance of the proposed algorithm (CCOCG) in the Internet node mobility management, cloud service perception, and WSNs opportunity communication and other environmental performance.

In the experiment, the Internet covering model of time domain, frequency domain, and spatial domain is considered. Random mobile model is adopted for the nodes and sensor nodes in the Internet. The cloud devices of cloud platform have the same configuration and deploy evenly. The basic parameters of the integrated network and WSN network are as follows: the maximum communication radius of nodes is $250 \mathrm{~m}$, and the fusion network size is $800 \mathrm{~m} \times 900 \mathrm{~m}$. The average value of the results of the 50 experiments was used as the final experimental results.

In the experiment, we compared and analyzed the performance of the proposed algorithm and VOR algorithm [19].
In the comparison of the experimental results, the change of the distance between two extremes and the sensor is changed, and the effect of the algorithm is analyzed.

When the number of the Internet nodes and the number of sensor nodes are different or equal, the coverage performance of the proposed algorithm and the VOR algorithm have been shown in Figure 5. The experimental results are as follows.

(1) With the increase of the size of nodes, the coverage area of the two algorithms is increased.

(2) When the number of nodes and the number of sensor nodes are different, the coverage performance of the proposed algorithm is better than the VOR algorithm. Because the proposed algorithm uses the opportunity sensor coverage method, the local node coverage information and the global coverage of WSN can be deployed and connected with the Internet to achieve global optimal coverage.

(3) With the increase of the number of iterations, the proposed algorithm based on cloud service area has a stronger coverage restoration and stable full connectivity.

With the different node of the mobile speed of the Internet, which executed 30 iterations and recover update, the coverage of the 2 algorithms of the full connectivity ratio is shown in Figure 6. The proposed algorithm provides a transparent interactive platform through the cloud platform to build a middleware layer between the Internet and WSN. There is mapping relationship between the network nodes and sensor nodes, which weaken the influence of the node moving speed and communication distance on the whole network. And the nodes can adaptively adjust the moving speed with the help of the cooperative cloud service sensing strategy. The network algorithm of full connectivity ratio was significantly higher 


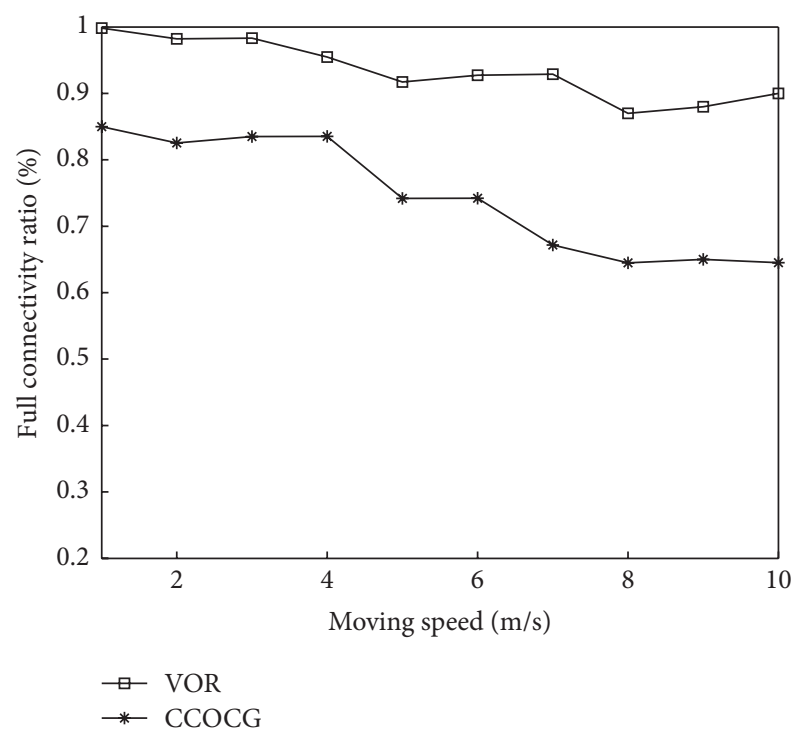

FIGURE 6: Full connectivity ratio.

than that in the VOR algorithm, and, in highly mobile nodes request, still has high communication rate.

\section{Conclusions}

How to improve the whole connectivity of the Internet through the integration of cloud platform and WSNs becomes an important technology of Internet application development. First, we studied the sensor opportunistic coverage mechanism based on the scale of Internet nodes, cloud, and sensor nodes. Second, according to the requirements of users and cloud platform computing ability, the cooperative cloud service aware model was given. Third, mobile Internet coverage connectivity guarantee protocol was proposed for resolving the mobility management, resource management and reliable information forwarding, and so forth. Mathematical analysis and simulation verification proved that the proposed scheme is superior to the VOR algorithm, such as coverage rate and full connectivity ratio, as well as connectivity guarantee price.

\section{Conflict of Interests}

The authors declare that there is no conflict of interests regarding the publication of this paper.

\section{Acknowledgments}

This work is supported in part by Key Scientific Research Project of Henan Province (15A520054) and Science and Technology Project of Henan Province (112102310550).

\section{References}

[1] B. Xie and A. Kumar, "A protocol for efficient Bi-directional connectivity between ad hoc networks and internet," Journal of Internet Technology, vol. 6, no. 1, pp. 101-108, 2005.

[2] N. Bartolini, G. Bongiovanni, T. F. La Porta, and S. Silvestri, "On the vulnerabilities of the virtual force approach to mobile sensor deployment," IEEE Transactions on Mobile Computing, vol. 13, no. 11, pp. 2592-2605, 2014.

[3] A. Sathiaseelan and J. Crowcroft, "Internet on the move: challenges and solutions," Computer Communication Review, vol. 43, no. 1, pp. 51-55, 2013.

[4] X. Ma, Y. Wang, and W. Sun, "Feverfew: a scalable coveragebased hybrid overlay for internet-scale pub/sub networks," Science China Information Sciences, vol. 57, no. 5, pp. 1-14, 2014.

[5] Z. Zheng, P. Sinha, and S. Kumar, "Sparse WiFi deployment for vehicular internet access with bounded interconnection gap," IEEE/ACM Transactions on Networking, vol. 20, no. 3, pp. 956969, 2012.

[6] X. Huang and J. Chen, "Efficient node coverage scheme of wireless sensor network under multi-constraint conditions," Journal of Networks, vol. 8, no. 10, pp. 2277-2284, 2013.

[7] S. Savazzi, V. Rampa, and U. Spagnolini, "Wireless cloud networks for the factory of things: connectivity modeling and layout design," IEEE Internet of Things Journal, vol. 1, no. 2, pp. 180-195, 2014.

[8] S. G. Hong, S. Seo, H. Schulzrinne et al., "ICOW: internet access in public transit systems," IEEE Communications Magazine, vol. 53, no. 6, pp. 134-141, 2015.

[9] M. Panda and D. P. Mohapatra, "Generating test data for path coverage based testing using genetic algorithms," in Proceedings of International Conference on Internet Computing and Information Communications, vol. 216 of Advances in Intelligent Systems and Computing, pp. 367-379, Springer, New Delhi, India, 2014.

[10] D. Zhao, H. Ma, L. Liu, and X.-Y. Li, "Opportunistic coverage for urban vehicular sensing," Computer Communications, vol. 60, pp. 71-85, 2015.

[11] V. M. Rohokale, S. Inamdar, N. R. Prasad, and R. Prasad, "Energy efficient four level cooperative opportunistic communication for wireless personal area networks (WPAN)," Wireless Personal Communications, vol. 69, no. 3, pp. 1087-1096, 2013.

[12] S. Misra, T. Ojha, and A. Mondal, "Game-theoretic topology control for opportunistic localization in sparse underwater sensor networks," IEEE Transactions on Mobile Computing, vol. 14 , no. 5, pp. 990-1003, 2015

[13] R. Tynan, C. Muldoon, G. O'Hare, and M. O’Grady, “Coordinated intelligent power management and the heterogeneous sensing coverage problem," Computer Journal, vol. 54, no. 3, pp. 490-502, 2011.

[14] Q. Qi, J. Liao, and Y. Cao, "Cloud service-aware location update in mobile cloud computing," IET Communications, vol. 8, no. 8, pp. 1417-1424, 2014.

[15] S. Nizamani and A. Kumari, "A quality-aware computational cloud service for computational modellers," Communications in Computer and Information Science, vol. 414, pp. 184-194, 2014.

[16] L. Chunlin and L. LaYuan, "Cost and energy aware service provisioning for mobile client in cloud computing environment," The Journal of Supercomputing, vol. 71, no. 4, pp. 1196-1223, 2015. 
[17] S. S. Savas, F. Dikbiyik, M. F. Habib, M. Tornatore, and B. Mukherjee, "Disaster-aware service provisioning with manycasting in cloud networks," Photonic Network Communications, vol. 28, no. 2, pp. 123-134, 2014.

[18] W. Dou, X. Zhang, J. Liu, and J. Chen, "HireSome-II: towards privacy-aware cross-cloud service composition for big data applications," IEEE Transactions on Parallel and Distributed Systems, vol. 26, no. 2, pp. 455-466, 2015.

[19] B. Wang, J. Qu, X. Wang, G. Wang, and M. Kitsuregawa, "VGQVor: extending virtual grid quadtree with Voronoi diagram for mobile $k$ nearest neighbor queries over mobile objects," Frontiers of Computer Science, vol. 7, no. 1, pp. 44-54, 2013. 

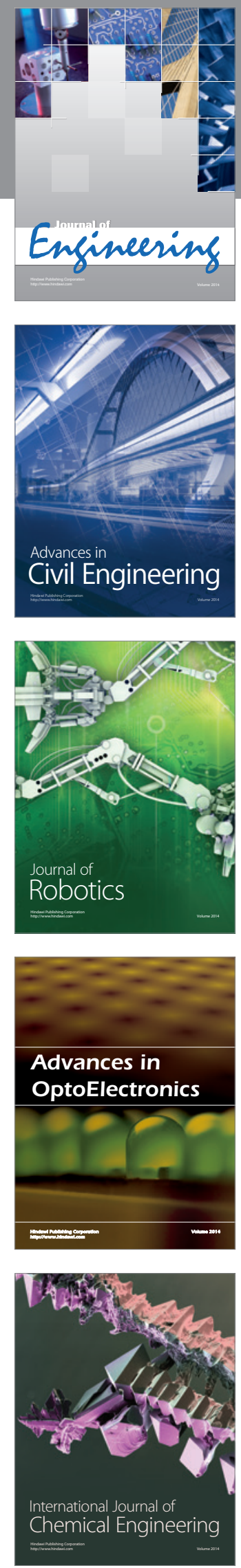

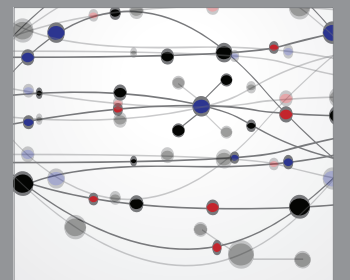

The Scientific World Journal
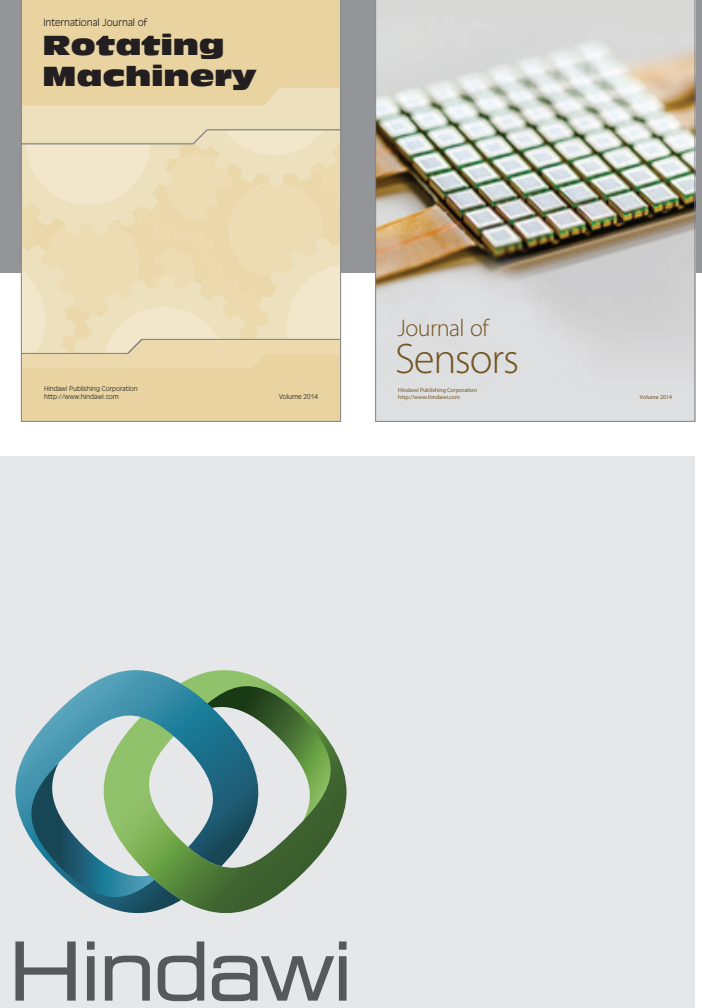

Submit your manuscripts at http://www.hindawi.com
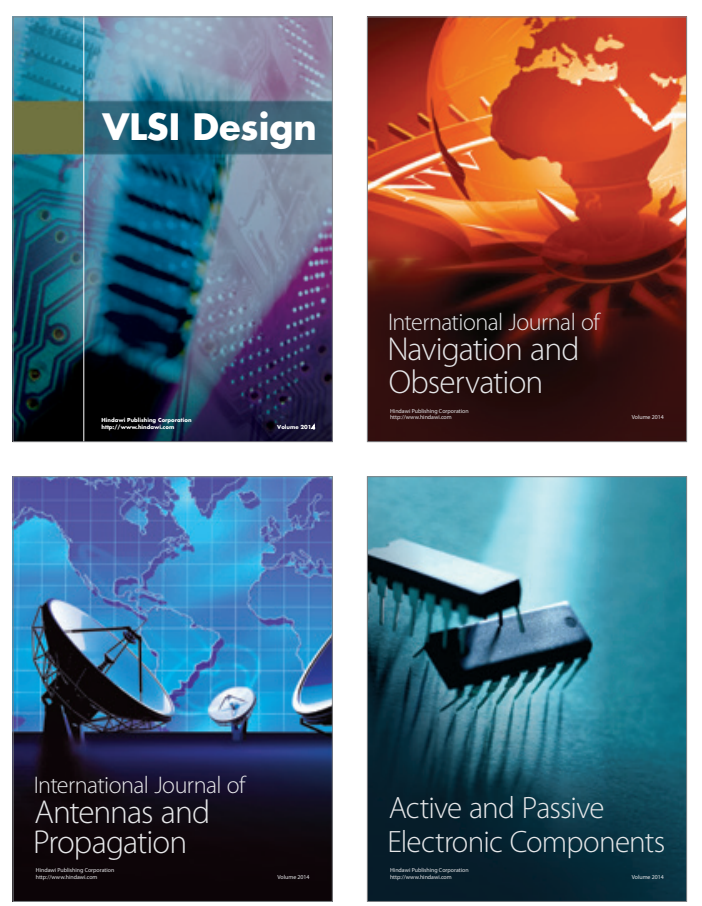
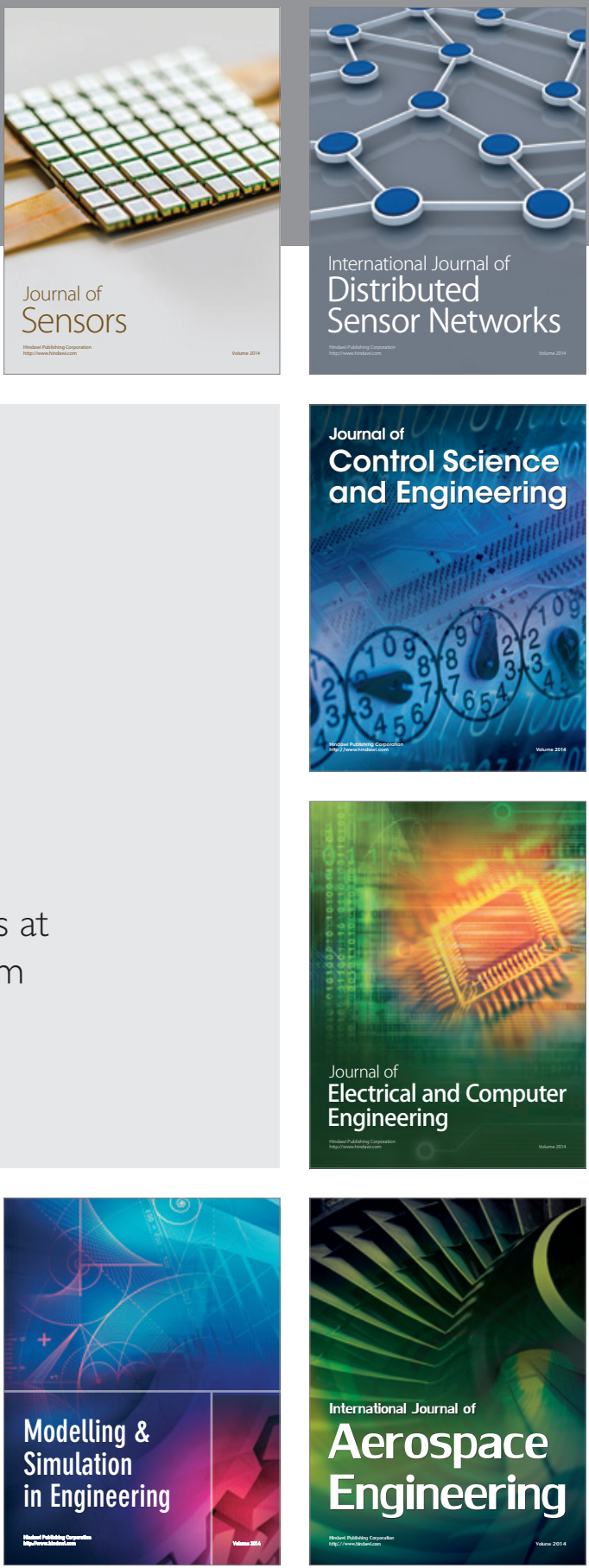

Journal of

Control Science

and Engineering
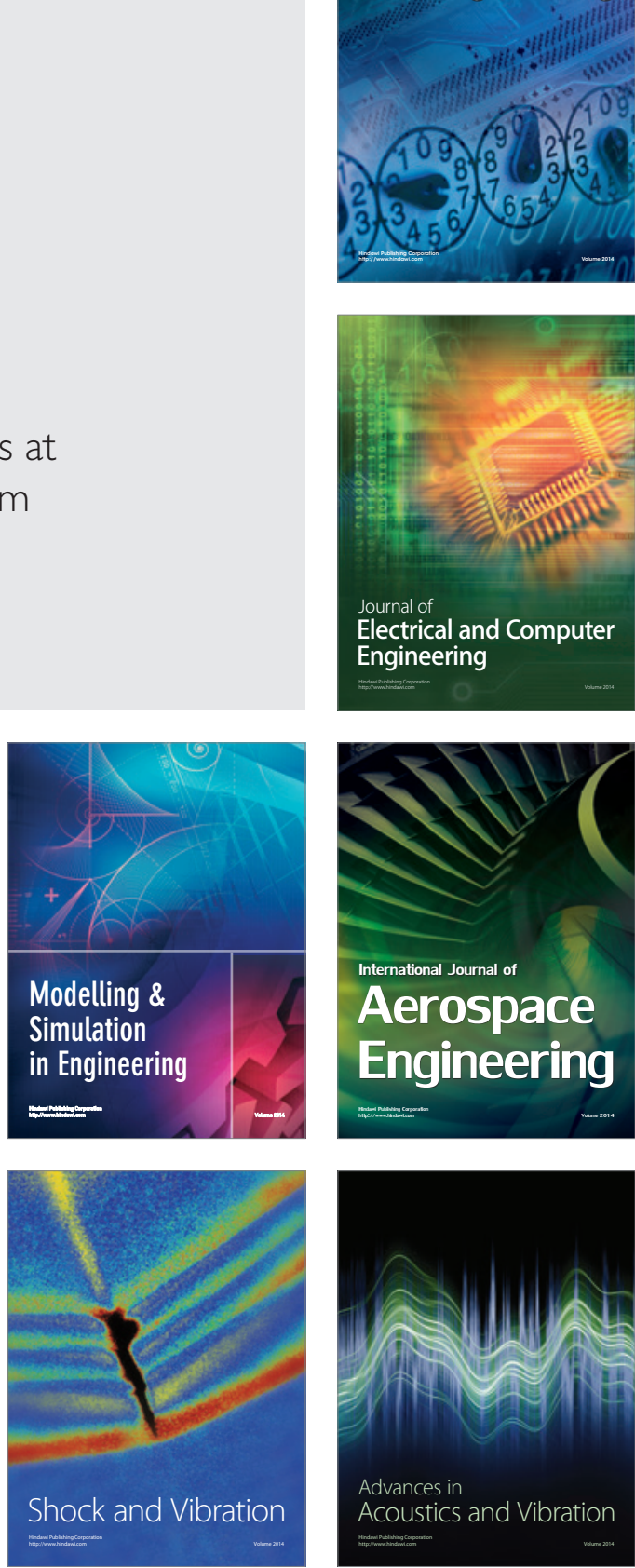